\title{
Patient-specific Parameterization of a Left-ventricular Model of Cardiac Electrophysiology Using Electrocardiographic Recordings
}

\author{
Karli Gillette ${ }^{1}$, Anton Prassl ${ }^{1}$, Jason Bayer ${ }^{2}$, Edward J. Vigmond ${ }^{2}$, Aurel Neic ${ }^{1}$, Gernot Plank ${ }^{1}$ \\ ${ }^{1}$ Institute of Biophysics, Medical University of Graz, Graz, Austria \\ ${ }^{2}$ LIRYC Electrophysiology and Heart Modeling Institute, Bordeaux Foundation, Pessac, France
}

\begin{abstract}
Introduction: Image-based models of human electrophysiology (EP) are increasingly considered as a clinical research tool. However, current clinical EP models are typically not patient-specific as they mostly rely on generic data or are computationally not efficient enough to fit with clinical time scales.

Objectives: This study aimed to develop an efficient, clinically-compatible automated workflow for patientspecific parameterization of cardiac EP models using noninvasive standard ECG recordings. Specifically, we focused on the parameterization of the depolarization phase during sinus activation to reproduce QRS morphology.

Methods: Two MRI-based left-ventricular (LV) models, A and $B$, were utilized. A simplified activation model was defined based on the assumption that activation patterns are determined by the locations, $\mathbf{x}$, of septal, anterior and posterior fascicle of the His-Purkinje system (HPS), their relative activation timings, $\mathbf{t}$, and the conduction velocities, $\mathbf{v}$, within $L V$ wall and HPS. A reaction-eikonal model was employed to compute activation sequences, source distributions and ECGs. Latin hypercube sampling was used to sweep the input parameter space $[\mathbf{x}, \mathbf{t}, \mathbf{v}]$. Quantitative comparison between $Q R S$ complexes of simulated and measured ECGs was performed using a normalized correlation coefficient and L2 norm.
\end{abstract}

Results: Activation sequences with corresponding QRS complex were simulated in approximately 25 seconds. Inherent morphological characteristics of the QRS complex could be represented by our model parameter space [ $\mathrm{x}$, $\mathbf{t}, \mathbf{v}$ ]. Correlation coefficients and L2 norms of 0.86 and 20.22 were attained for model $A$, and 0.93 and 3.06 for model $B$, respectively.

Discussion: The feasibility of generating patient-specific $L V$ activation sequences based on measured QRS complexes in non-invasive ECG recordings was demonstrated. The efficiency of the proposed model will facilitate its use in a future more general framework for data-driven clinical EP model parameterization.

\section{Introduction}

Image-based models of human cardiac electrophysiology (EP) have been extensively used to understand the mechanisms behind cardiac arrhythmias. With recent advancements in model generation and simulation, cardiac EP models have also become increasingly viable as a clinical tool for personalized diagnostics and treatment of arrhythmias leading to increased prognosis [1,2].

These models generally implement standardized cardiac EP or require additional patient data that is invasive to be generated in clinical times scales, and therefore often entirely neglect a patient's specific EP [2]. Only a few number of studies have attempted to achieve functional EP specificity in a clinical context [3]. Zettinig, et.al. 2014 [3], for example, uses data-driven model estimation to fit parameters of a computational model based on the 12 lead ECG. However, this study is limited in scope by using only QRS duration and electrical axis deflection as resultant patient personalization. It is therefore necessary to develop clinically viable workflow for direct EP model personalization matching to a full set of non-invasive EP recordings, such as the 12 lead ECG, that is capable of exploring a large parameter space.

This study aimed to generate a computationally efficient workflow for EP model personalization based on non-invasive ECGs. In particular, an MRI-based, leftventricular (LV) model of cardiac EP was automatically parameterized to generate patient-specific ventricular depolarization matching the QRS complex of corresponding ECGs taken from a 12 lead ECG. Two approaches of automatic parameterization were utilized, piecewise and full, to explore how input parameters influence resultant simulated QRS morphology. We build upon the work presented in Augustin, et. al 2016 [4], which used manual parameterization towards the same end.

\section{Methods}

Comprehensive clinical datasets acquired from a cohort of 110 pediatric patients suffering from aortic valve dis- 
ease were used for building a set of twenty anatomically accurate finite element models of the LV [4]. Two cases B0504-44 and B0399-47 with corresponding ECG recordings of lead I, II, and V1 during sinus rhythm were selected. Models were equipped with rule-based fiber architecture [5] and partitioned into nine regions using algebraic combinations of laplacian and eikonal gradient solutions to facilitate the assignment of transmural and apico-basal EP heterogeneities, respectively [6]. Regional cellular dynamics in each region were represented by the tenTusscherNoble-Noble-Panfilov model [7] with region-specific adjustments [8].

Impulse propagation on the LV endocardium was initiated at the assumed locations of the fascicular terminals, $\mathbf{x}$, corresponding to the septal $\mathbf{x}_{\mathrm{sf}}$, anterior, $\mathbf{x}_{\mathrm{af}}$, and posterior, $\mathbf{x}_{\mathrm{pf}}$, fascicles. For each fascicle, both the timings, $\mathbf{t}$, and radii of direct activation, $\mathbf{r}$, were prescribed.

The network topology of the His-Purkinje system (HPS) lining the LV endocardium and the locations of Purkinjeventricular junctions were not explicitly modeled. Rather, a subendocardial layer of faster $\mathrm{CV}, v_{\mathrm{ps}}$, was assumed as a surrogate that facilitates the fast spread activation over the LV endocardium. CVs in the LV were assumed to be orthotropic. The steerable input parameters vector, $\mathbf{q}$, that were explored is therefore given as $\mathbf{q}=$ $\left\{\mathbf{x}_{\mathrm{sf}}, \mathbf{x}_{\mathrm{af}}, \mathbf{x}_{\mathrm{pf}}, \mathbf{t}_{\mathrm{sf}}, \mathbf{t}_{\mathrm{af}}, \mathbf{t}_{\mathrm{pf}}, \mathbf{r}_{\mathrm{sf}}, \mathbf{r}_{\mathrm{af}}, \mathbf{r}_{\mathrm{pf}}, \mathbf{v}_{\mathrm{ps}}\right\}$.

Simulations were performed using CARP [9]. Specifically, a coupled reaction-Eikonal (R-E) model [8] was used to compute the activation sequence and electrical source distribution throughout the LV. Briefly, the R-E model is equivalent to a standard reaction-diffusion monodomain model given as

$$
\beta C_{\mathrm{m}} \frac{\partial V_{\mathrm{m}}}{\partial t}=\nabla \cdot \boldsymbol{\sigma}_{\mathrm{i}} \nabla\left\{V_{\mathrm{m}}\right\}+I_{\text {foot }}\left(t_{\mathrm{a}}\right)-\beta I_{\text {ion }}
$$

that is augmented with an artificial current, $I_{\text {foot }}$.

This current approximates the electrotonic current driving the foot of an action potential and is triggered at instants of wavefront arrival times computed by solving the Eikonal equation [10]. The key advantage of this implementation of a R-E model is the independency of computed source distributions upon spatial resolution thus enabling the use of coarser discretized and thus computationally much cheaper meshes as it would be feasible with plain reaction-diffusion models. The eikonal equations were solved using an advancing wavefront approach [11].

For simulating wavefront propagation the diffusion term was neglected, rendering the solution of Eq. (1) a localized problem [12]. 12-lead ECGs were then reconstructed from an artificial lead system by evaluating an integral solution of Poisson's equation assuming that the LV is immersed in an infinite unbounded medium of $1 \mathrm{~S} / \mathrm{m}$ conductivity.
First, a latin hypercube of 2000 runs was used to sample combinations for model B0504-44 and B0399-47 across the entire input parameter space $\mathbf{q}$ and sweeping $v_{\mathrm{ps}}$ from 1 to $4 \mathrm{~m} / \mathrm{s}$. Given that each simulation could be temporally scaled, $t_{\mathrm{sf}}$ was initiated at zero. To investigate the influence of parameters, a second piecewise optimization was also performed. First, a latin hypercube sampled $\mathbf{q}$ for a subset of 1000 combinations for only $\mathbf{x}$ and $\mathbf{t}$. Simulations were conducted assuming a constant $v_{\mathrm{ps}}$ of $2.0 \mathrm{~m} / \mathrm{s}$ and $\mathbf{r}$ of $0.1 \mathrm{~mm}$ resulting in singular nodal activation. The stimulus parameters of $\mathbf{x}$ and $\mathbf{t}$ which provided the best ECG fit were passed to the next stage and swept for $v_{\mathrm{ps}}$. Third, a latin hypercube of 250 runs sampled $r_{\mathrm{sf}}, r_{\mathrm{af}}, r_{\mathrm{pf}}$ using the optimized stimuli and $v_{\mathrm{ps}}$.

Normalized correlation coefficients (CCs) and L2 norms served as quantitative metrics of fit between the QRS morphology of simulated and reference ECGs. A modified QRS detection algorithm was used for the alignment of the simulated QRS complex to the QRS complex of a single beat within lead I of the reference ECG [13]. Once aligned, the simulated ECGs were scaled to the global maximum and minimum of the reference ECG. The CCs and L2 norms were normalized across all three leads.

\section{Results}

Each simulation and analytical comparison for B039947 and B0504-44 lasted approximately 30s and 22s. Outcomes of both full and piecewise parameter optimization are summarized in Table 1 . The simulation setup and resultant activation waveform leading to QRS morphology for B0399-47 is shown in figure 2.

Table 1: Optimal input parameters for both B0504-44 and B0399-47 with corresponding result metrics. Locations are presented as apico-basal and rotational coordinates. Apico-basal ranges from 0 (apex) to 1 (base). Rotation ranges from -1 to 1 with 0 indicating the septal midline.

\begin{tabular}{ccccc} 
& \multicolumn{2}{c}{ Full } & \multicolumn{2}{c}{ Piecewise } \\
Parameter & B0504-44 & $\mathbf{B 0 3 9 9 - 4 7}$ & $\mathbf{B 0 5 0 4 - 4 4}$ & B0399-47 \\
\hline $\mathbf{x}_{\mathrm{sf}}$ & {$[0.49,0.55]$} & {$[0.78,0.85]$} & {$[0.63,0.97]$} & {$[0.39,0.7]$} \\
$\mathbf{x}_{\mathrm{af}}$ & {$[0.43,0.19]$} & {$[0.82,0.17]$} & {$[0.87,0.76]$} & {$[0.46,0.35]$} \\
$\mathbf{x}_{\mathrm{pf}}$ & {$[0.50,0.93]$} & {$[0.83,0.46]$} & {$[0.23,0.36]$} & {$[0.61,0.06]$} \\
$t_{\mathrm{af}}(\mathrm{s})$ & 7.07 & 5.76 & 8.98 & 6.47 \\
$t_{\mathrm{pf}}(\mathrm{s})$ & 5.01 & 0.92 & 4.18 & 6.41 \\
$r_{\mathrm{sf}}(\mathrm{mm})$ & 1.60 & 2.03 & 0.10 & 0.10 \\
$r_{\mathrm{af}}(\mathrm{mm})$ & 4.99 & 1.34 & 0.10 & 0.10 \\
$r_{\mathrm{pf}}(\mathrm{mm})$ & 3.58 & 1.74 & 0.10 & 0.10 \\
$v_{\mathrm{ps}}(\mathrm{m} / \mathrm{s})$ & 1.7 & 3.1 & 2.0 & 2.3 \\
\hline time $(\mathrm{hrs})$ & 14.15 & 19.09 & 8.21 & 10.54 \\
CC & 0.86 & 0.93 & 0.92 & 0.80 \\
L2 norm & 20.22 & 3.06 & 11.35 & 6.99 \\
\hline
\end{tabular}

Resultant QRS morphology alongside reference ECGs, are shown for both models and optimization methods in 

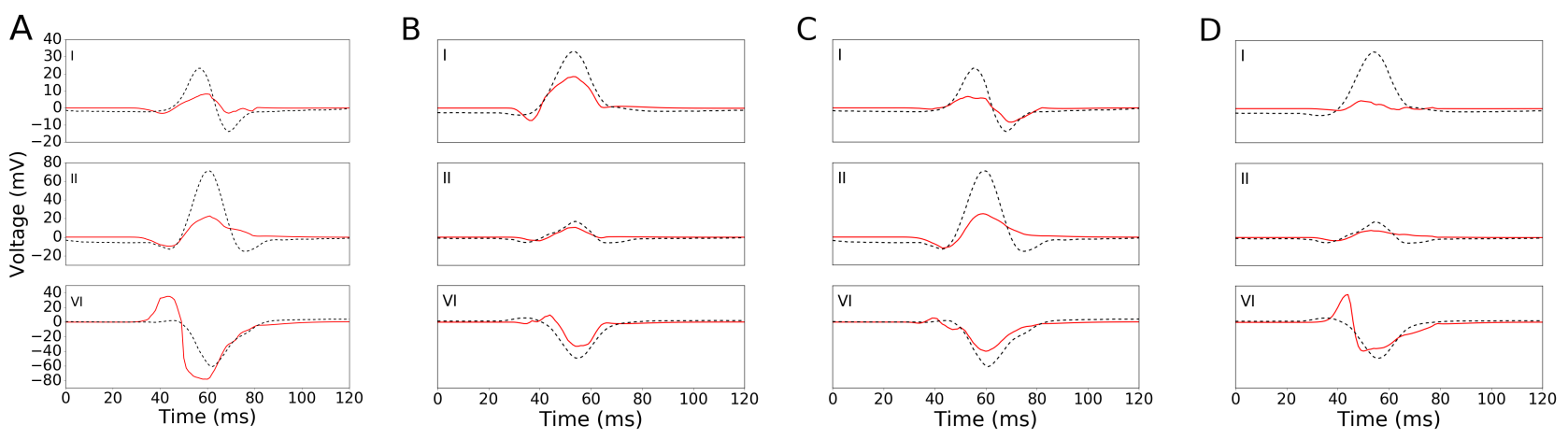

Figure 1: Simulated (red) and reference (black) QRS morphology for leads I, II, and V1 of model B0504-44 (A,C) and model B0399-47 (B,D) for full (A,B) and piecewise (C,D) optimizations. Vertical axis is applied across all subfigures.

figure 1. General morphological curvature of the reference QRS complexes could be recreated (Figure 1) using the proposed input parameter space $\mathbf{q}$ for both models, but with the highest observed fit in Figure 1b. Fractionation can be observed in the simulated QRS morphology, along with dampened amplitudes primarily in leads I and II.

Full-parameter optimization required longer computation times due to an increased number of required simulations, but led to drastically better quantitive metrics (Table 1) and QRS morphology (Figure 1b) for B0399-47. In model B0504-44, higher quantitative metrics were attained using piecewise optimization leading to somewhat better QRS morphology (Figure 1c). Piecewise parameterization revealed alteration of $\mathbf{r}$ following stimulus site optimization did not result in improved metrics in either model.

\section{Discussion}

This study demonstrated the generation of patientspecific ventricular depolarization sequences in an imagebased LV model of cardiac EP according to ECGs during sinus rhythm within clinical time scales. The proposed workflow serves as a technical starting point for additional advancements and implementations of EP personalization of image-based models of cardiac EP using non-invasive EP recordings like the 12 lead ECG. It is our intent to increase efficiency of the proposed workflow using advanced computer automation techniques to allow for optimization of larger parameter spaces on more anatomically specific models while still remaining clinically relevant.

The LV model was reduced to 8 parameters relating to timing, radii, and location of three stimulus sites, along with maximal $\mathrm{CV}$ in the subendocardial layer. Additional parameters and can be introduced to generate potentially better fits using full-scale parameterization as was possible for B0399-47. For example, higher patient speci- ficity could be achieved by including a fully characterized HPS by mapping pre-constructed topological networks or through the utilization of a cardiac conduction system algorithm [14].

A larger parameter space, however, inherently requires increased computational time and cost. While it appears that a reduced input parameter space of $\left\{\mathbf{x}_{\mathrm{sf}}, \mathbf{x}_{\mathrm{af}}, \mathbf{x}_{\mathrm{pf}}, \mathbf{t}_{\mathrm{sf}}, \mathbf{t}_{\mathrm{af}}, \mathbf{t}_{\mathrm{pf}}, \mathbf{v}_{\mathrm{ps}}\right\}$ could adequately recreate QRS morphology, such results could merely be a function of latin hypercube sizing. Convergence on a local optimum, as is probable in the full parameterization of B0504-44, could be avoided by investigating the number of simulations required to achieve adequate fits. Computational cost of optimization of large parameter spaces could instead be reduced by implementing sampling methods with better space-filling properties, iterative parameter feedback, or machine learning approaches.

More realistic and fully characterized data sets are a necessary extension of this study. Data sets that include a corresponding torso anatomy and complete 12 lead ECGs would allow better forward approximations of simulated ECGs and more thorough analysis of optimization within the confines of the current framework. Complete ECG morphology could be simulated and matched using four chamber heart models.

\section{Acknowledgements}

This research was supported by the Grant F3210- N18 from the Austrian Science Fund, and the European Commission Grant CardioProof 611232.

\section{References}

[1] Trayanova NA, Chang KC. How computer simulations of the human heart can improve anti-arrhythmia therapy. The Journal of physiology 2016; 

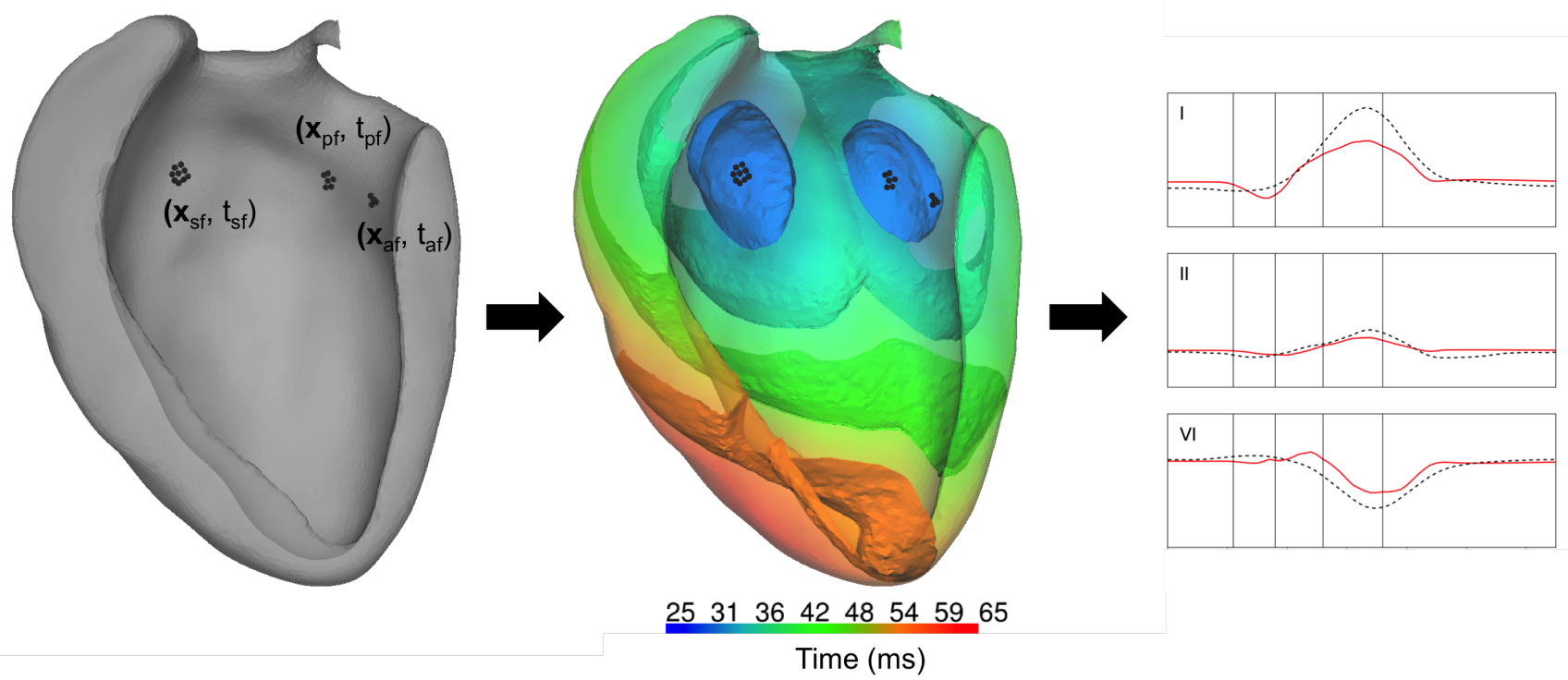

Time (ms)

Figure 2: Stimulation setup (left) and resultant ventricular depolarization (middle) sequence for B0399-47 using parameters acquired during full parameterization. Activation isochrones correspond to vertical lines in simulated ECG.

[2] Arevalo HJ, Vadakkumpadan F, Guallar E, Jebb A, Malamas P, Wu KC, Trayanova NA. Arrhythmia risk stratification of patients after myocardial infarction using personalized heart models. Nature communications 2016;7.

[3] Zettinig O, Mansi T, Neumann D, Georgescu B, Rapaka S, Seegerer P, Kayvanpour E, Sedaghat-Hamedani F, Amr A, Haas J, et al. Data-driven estimation of cardiac electrical diffusivity from 12-lead ecg signals. Medical image analysis 2014;18(8):1361-1376.

[4] Augustin CM, Crozier A, Neic A, Prassl AJ, Karabelas E, da Silva TF, Fernandes JF, Campos F, Kuehne T, Plank G. Patient-specific modeling of left ventricular electromechanics as a driver for haemodynamic analysis. Europace 2016; 18(suppl 4):iv121-iv129.

[5] Bayer J, Blake R, Plank G, Trayanova N. A novel rulebased algorithm for assigning myocardial fiber orientation to computational heart models. Annals of biomedical engineering 2012;40(10):2243-2254.

[6] Keller DU, Weiss DL, Dossel O, Seemann G. Influence of heterogeneities on the genesis of the t-wave: A computational evaluation. IEEE Transactions on Biomedical Engineering 2012;59(2):311-322.

[7] Ten Tusscher K, Noble D, Noble P, Panfilov A. A model for human ventricular tissue. American Journal of Physiology Heart and Circulatory Physiology 2004;286(4):H1573H1589.

[8] Neic A, Campos FO, Prassl AJ, Neiderer SA, Bishop MJ, Vigmond EJ, Plank G. Efficient computation of electrograms and ecgs in human whole heart simulations using a reaction-eikonal model, 2017.
[9] Vigmond EJ, Hughes M, Plank G, Leon LJ. Computational tools for modeling electrical activity in cardiac tissue. Journal of electrocardiology 2003;36:69-74.

[10] Colli Franzone P, Guerri L, Rovida S. Wavefront propagation in an activation model of the anisotropic cardiac tissue: asymptotic analysis and numerical simulations. Journal of mathematical biology 1990;28(2):121-176.

[11] Fu Z, Jeong WK, Pan Y, Kirby RM, Whitaker RT. A fast iterative method for solving the eikonal equation on triangulated surfaces. SIAM Journal on Scientific Computing 2011;33(5):2468-2488.

[12] Bishop MJ, Plank G. Bidomain ecg simulations using an augmented monodomain model for the cardiac source. IEEE transactions on biomedical engineering 2011; 58(8):2297-2307.

[13] Pan J, Tompkins WJ. A real-time qrs detection algorithm. IEEE transactions on biomedical engineering 1985; (3):230-236.

[14] Sebastian R, Zimmerman V, Romero D, Frangi AF. Construction of a computational anatomical model of the peripheral cardiac conduction system. IEEE Transactions on Biomedical Engineering 2011;58(12):3479-3482.

Address for correspondence:

Gernot Plank

Institute of Biophysics, Medical University of Graz, Harrachgasse 21, 8010, Graz, Austria

gernot.plank@medunigraz.at 